\title{
A geospatial database management system for the collection of medicinal plants
}

\author{
Supattra Puttinaovarat, ${ }^{1}$ Paramate Horkaew ${ }^{2}$ \\ ${ }^{1}$ Faculty of Science and Industrial Technology, Prince of Songkla University, Surat Thani Campus, Surat \\ Thani; ${ }^{2}$ School of Computer Engineering, Institute of Engineering, Suranaree University of Technology, \\ Nakhon Ratchasima, Thailand
}

\begin{abstract}
Medicinal plants are increasingly used, both for medical applications and personal healthcare. However, existing herbal database systems for plant retrieval offer only basic information and do not support real-time analysis of the spatial aspects of plantations and distribution sites. Moreover, data records are usually static and not publicly available as they rely on costly proprietary software packages. To address these shortcomings, including limiting the time needed for collection and data processing, a novel medicinal plants geospatial database management system is proposed. The system allows localization of plant sites and data presentation on an interactive heat map displaying spatial information of plants selected by the user within a specific radius from the user's location, including automatic presentation of an itinerary giving the optimal route between user location and plant destinations selected. The approach relies on dynamic and role-based data management, an interactive map that includes graphics and integrated geospatial analyses thanks to cross-platform, geograph-
\end{abstract}

Correspondence: Paramate Horkaew, School of Computer Engineering, Institute of Engineering, Suranaree University of Technology, Nakhon Ratchasima 30000, Thailand.

E-mail: phorkaew@sut.ac.th ; paramate@gmail.com

Key words: Medicinal plants; geospatial database; web application; geographical information system; Thailand.

Acknowledgements: the authors are grateful to Surat Thani provincial public health office for providing research grant for the studies and development of the presented system. We also thank Surat Thani provincial agriculture and commerce offices and Prince of Songkla University, Surat Thani campus, for providing valuable suggestions, requirements and medicinal plant data, employed in this study.

Contributions: the authors contributed equally to this work.

Received for publication: 12 March 2021

Revision received: 29 April 2021.

Accepted for publication: 29 April 2021.

(C) Copyright: the Author(s), 2021

Licensee PAGEPress, Italy

Geospatial Health 2021; 16:998

doi:10.4081/gh.2021.998

This article is distributed under the terms of the Creative Commons Attribution Noncommercial License (CC BY-NC 4.0) which permits any noncommercial use, distribution, and reproduction in any medium, provided the original author(s) and source are credited. ical a JavaScript library and Google API. Both spatial data and attributes are available in real time. The system would support effective collaboration, among herb farmers, government agencies, private investors, healthcare professionals and the general public with regard to various aspects of medicinal plants and their applications.

\section{Introduction}

In Thailand, the Ministry of Public Health promotes the use of medicinal plants in the community as a means of relieving symptoms, complementary to modern medicine (Chotchoungchatchai et al., 2012; Ochwang'i et al., 2017). Farmers and the general public both grow and consume these herbs (Kolasinski, 2014; Serafini and Peluso, 2016). Nonetheless, due to ineffective public relation approaches, many consumers, pharmaceutical companies and government agencies have limited access to information about the distribution of valuable medicinal plants and commercial plantations, thus undermining their potential. Such plants have been used for manufacturing herbal medicines, instant food and beverages and cosmetics (Khaerunnisa et al., 2020). A system equipped with geospatial visualization can help determining whether the growth of desired medicinal plants is dense enough to allow largescale production. Moreover, it can assist finding the nearest plantation locations where the plants are grown.

Medicinal plant information systems can be categorized according to two foci: i) retrieval of data on therapeutic use; and ii) development of herbal plant database applications. The former includes creation of knowledge bases matching diseases with their herbal remedies (Ogirima, 2015; Tungkwampian et al., 2015; Fausat et al., 2019). For instance, users can look up plant names, their medicinal properties, their use for curing diseases and other relevant details, e.g. suitable climates for plantation or ontology techniques in designing a database for therapeutic information retrieval (Tungkwampian et al., 2015). The latter deals with the development of herbal plant database applications allowing users to ask questions on the details of specified medicinal plants (Mary et al., 2012; Chantraket et al., 2014; Mashayekhan et al., 2016; Biswas et al., 2017; Bultum et al., 2019). Relational database management system (RDBMS) is often employed for storing primarily descriptive and graphical data (Mary et al., 2012; Bultum et al., 2019); however, they lack spatial capability, e.g. Medherb (Mason, 2002), HerbMed (Wootton, 2002), HerbMedPro (Saxton and Owen, 2005) and InPACdb (Vetrivel et al., 2009). Later developments of online software packages have proposed herbal plant information management web applications managing names of herbs, their medicinal properties and images overcoming previous limitation that only allowed authorized access to data management functions (Mary et al., 2012). Since these databases compile data from various sources, including research papers and books, their 
contribution was much more extensive. However, a major problem was that online user participation was not permitted (Bultum et al., 2019). In addition, given merely descriptive data, users can learn only general data on the herbal plants of interest, with plantations and distribution sites broadly expressed by standard address fields (e.g. village, district, etc.), instead of exact geographical locations.

A number of studies have applied different versions of geographical information systems (GIS) when developing spatial herbal plant databases and web applications (Chantraket et al., 2014; Mashayekhan et al., 2016; Biswas et al., 2017). One of the earliest developments was a prototype web application for medicinal plants in Phatthalung Province, Thailand (Jungialern et al., 2012). It was based on QGIS (https://www.qgis.org/en/site/), a full-featured, user-friendly, open-source GIS. In doing so, the authors also used PostgreSQL (https://www.postgresql.org/) and POSTGIS (https://postgis.net/), two other open-source applications where the latter adds support for geographic objects to the PostgreSQL object-relational database. The main drawback of this approach is the absence of search and report functions since it was a static web application that did not offer data management online. Another static, spatial herbal database system based on ArcGIS server and ArcSDE (ESRI, Redlands, CA, USA) was proposed by Chantraket et al. (2014) but it is relatively costly and also lacks information on herbal plantations, potential products and distribution sites. A third, static map application was proposed by Mashayekhan et al. (2016) who aimed at locating plantations of specified herbal plants; however, the approach suffered from similar limitations. Biswas et al. (2017) adopted the ArcGIS's online spatial data management platform that allows developers to incorporate their own data via a web browser, thereby extending the premade functions without having to write the entire software. Nonetheless, even if this approach is good and also free of charge, the free period lasts only for a limited period, with significant charges incurred in the long run. Moreover, only the administrator, not the general user, is authorized to make changes of stored data. Another early GIS study presented by Al-Bakri et al. (2011) in Jordan addressed the spatial analysis of herbal plant distribution sites focusing on individual herbs and plantations, but like other static map systems the program is unable to dynamically update data already recorded. More recent research (Wu et al., 2019) employed ArcGIS and implemented, with Python language, a machine learning model investigating suitable ecology system for planting medicinal plants. The factors considered in this approach were climate, soil data, plantation distribution, administrative boundaries and urban areas.

The major shortcomings impeding successful and efficient applications of spatial analyses of question concerning medicinal plant of currently available applications are that they only account for descriptive data, the static databases used exclude both update by users and extension to other regions, while conditional, spatial queries cannot be made. The Thai Government has initiated a pilot project aimed at dissemination and presentation to the general public of information related to medicinal plants that includes herbal plantation, product description, marketing and distributing channels, etc., but a computerized system organizing such information was lacking. Hence, alleviating the limitations described above, we wished to develop a geospatial database system based on modern web technologies, state-of-the-art GIS and spatial analytical libraries intended to produce integrated geospatial analyses together with an interactive graphical map with the main emphasis placed on efficient access to and potential usage of well systemized data.

\section{Materials and methods}

\section{Study area}

The study took place in Surat Thani located in the upper southern region of Thailand, where a variety of medicinal plants are found (Figure 1). This area is one of the pilot provinces participating in herbal plant cities project initiated by the Thai Government mentioned above.

\section{Data preparation}

In the experiments and subsequent development of a spatial medicinal plant database, we gathered within the study area the relevant data consisting of: i) herbal plants cultivated in the area, obtained from the Surat Thani Provincial Public Health Office; ii) herbal plantations obtained from the Surat Thani Provincial Agriculture Office; iii) herbal products and their distribution sites, obtained from Surat Thani Provincial Commerce Office; and iv) local herbal research, obtained from Prince of Songkla University, Surat Thani campus.

These data were stored in various digital formats, e.g. in MS Excel, MS Word and as imagery (stored in different static websites hosted by relevant agencies and institutes). The information was not only used for analysis and system design, but also as input for a spatial database system. The latter would then serve as preliminary 'data as a service', provided by the prototype system. Detailed descriptions are provided in Table 1. Note that, subject to their availability, data from other provinces might be incorporated into this system for extended services.

\section{Obligations}

In addition to reviewing limitations of existing systems, this research collected user requirements from relevant provincial agencies and academic institutes in Surat Thani, i.e. the Public Health Office, the Agriculture Office, the Commerce Office and the Prince of Songkla University, Surat Thani campus. The expected, required additional functions missing at the time we first engaged in this work were the following: i) presentation of herbal and relevant data, both in descriptive and spatial form; ii) availability of ways to manage and update the database (add, edit, delete) both by officers depending on role and level of authorization and

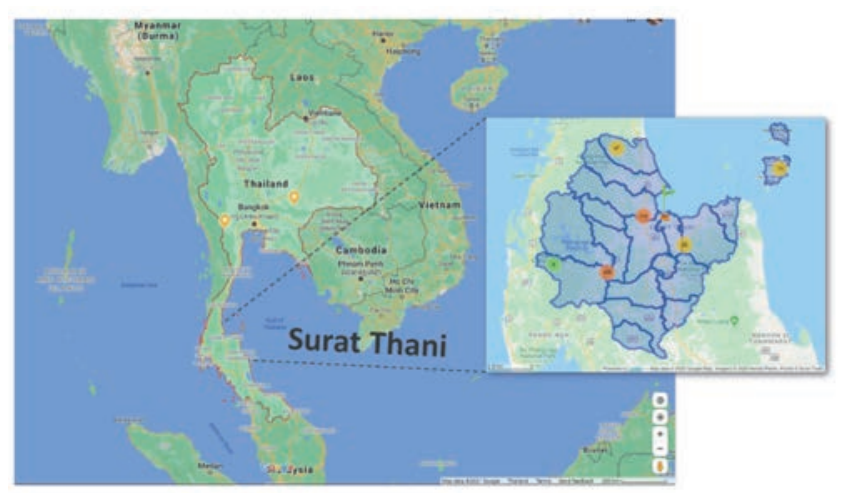

Figure 1. The Surat Thani study area in the upper southern region of Thailand. 
general users (i.e. officer, administrative staff, user); iii) possibility of conditional data access, both spatially and by the use of descriptive keys; and iv) option of managing both spatial data and analyses, e.g. address conversion into geographical coordinates and vice versa; applying distribution and density analyses of plantation and distribution sites; and automatic presentation of an itinerary between a user's location and sites of interest.

The use of the proposed system is depicted in Figure 2. As shown in this diagram, general users can access and retrieve herbal and relevant data both descriptively and spatially. They can also access a range of spatial functions, such as density growth mapping at herbal plantations; navigation from current location to a plantation or a distribution site; and finding graphical reports on sites of interest within a given radius from the user.

\section{System design}

The application is written in PHP language, using an opensource JavaScript Leaflet, an interactive map library (https://leafletjs.com/) that supports a number of extension plugins. Leaflet is easy to use, has a well-documented application programming interface (API), operates on both desktop and mobile platforms and, most importantly, is adaptable to other computerized GIS frameworks. Turf.js (https://turfjs.org/) was included for spatial data analysis and statistics. Descriptive and geographical herbal data were stored and managed by PostgreSQL and POSTGIS, respectively.

The application developed consists of 6 main modules addressing various concerns and limitations associated with existing systems. All modules support data analysis and presentation through an interactive map. The main characteristics of the new system are the following: i) the spatial data representation module, primarily driven by Leaflet, displays coordinates for both herbal plantations and distribution sites. Changes arising from data management are updated on the map in real time; ii) the geographical coordinate module is summarized in Figure 3A. It includes three steps, i.e. data input, pre-processing and transformation. The user first specifies the herbal plantation, which gives the latitude and longitude according to the universal transverse mercator (UTM) coordinate system, which can then be recorded in the geospatial database. If not found, the plantation address is searched by Geocode API and the result recorded. If both approaches fail, the user is notified by an error message. Figure 3B shows the screen shot of the interactive map for this with explanations; iii) the spatial analysis and presentation module searches herbal plantations, distribution sites and product data presenting them on a map where the user's location is shown (or any other site specified by the user). Centred at this location, a buffer contour circle defines zones by specified radii, e.g. $1 \mathrm{~km}$, to visualize aggregated data in this area. Buffers are processed and drawn by Turf.js, e.g. as seen in Figure 4; iv) the rout-

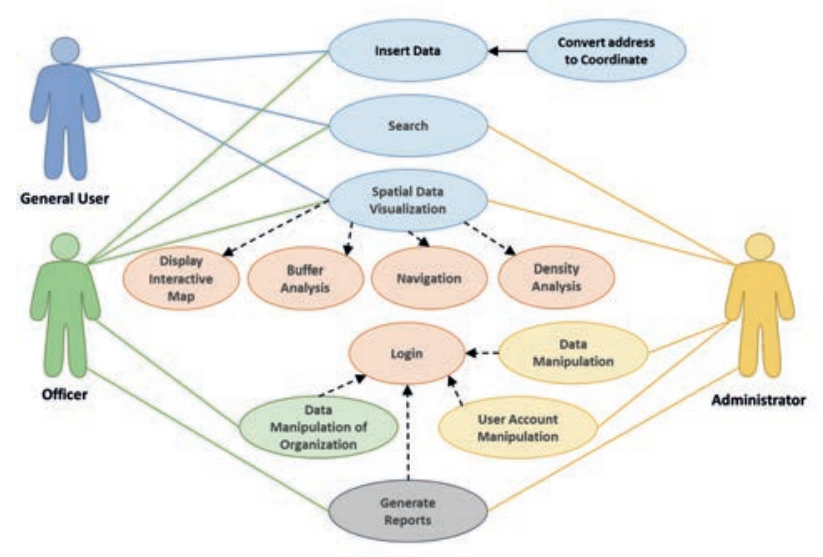

Figure 2. Diagram of the system consisting of the three user groups: general user, officers and administrator.

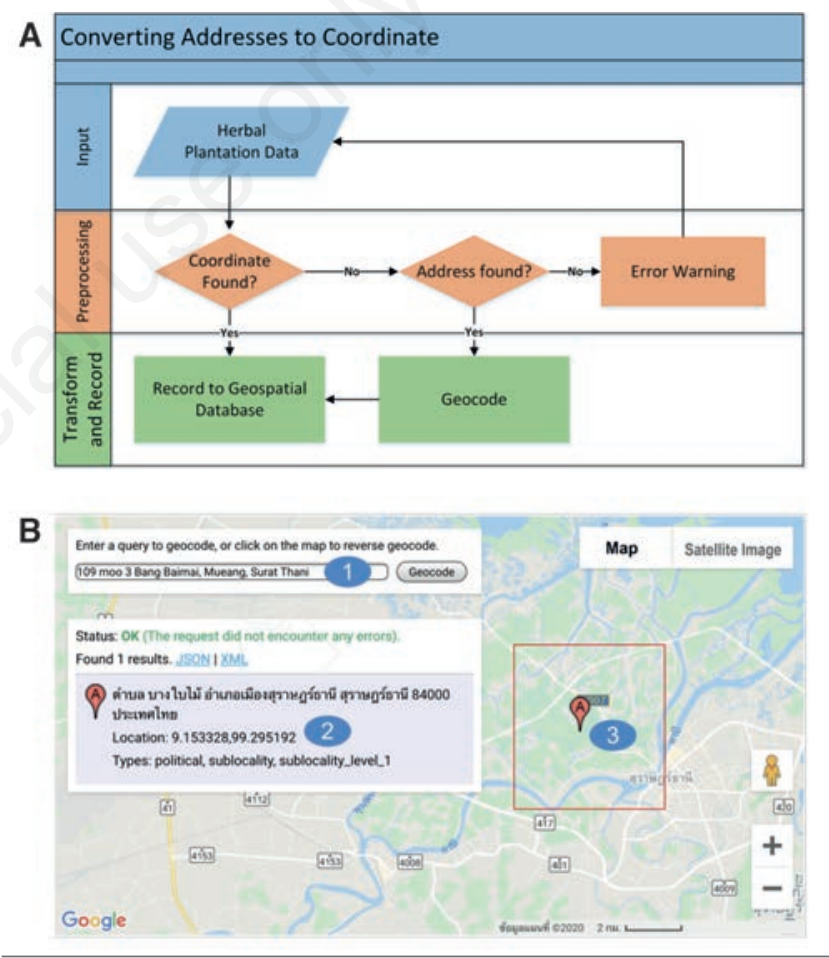

Figure 3. Finding and recording address and the corresponding latitude and longitude: A) steps taken when addressing the coordinate conversion module; B) screenshot of the interactive map, where (1) indicates the text box (under the graphical user interface (GUI) control) for input of the plantation address; If found, the corresponding coordinates are listed in the box below (2) and pinned on the map (3).

Table 1. Detailed descriptions of the input herbal plant data considered in this study.

\begin{tabular}{lcc} 
Data & Records (no.) & Organivation \\
$\begin{array}{l}\text { General information } \\
\text { Herbal plantation }\end{array}$ & 68 & $\begin{array}{c}\text { Surat Thani provincial public health office } \\
\text { Surat Thani provincial agriculture office }\end{array}$ \\
\hline $\begin{array}{l}\text { Herbal products } \\
\text { Herbal distribution }\end{array}$ & 37 & Surat Thani provincial commerce office \\
& 20 & Surat Thani provincial commerce office \\
\hline Herbal research & 5 & Prince of Songkla University, Surat Thani Campus
\end{tabular}




\section{Results}

tion sites by drawing the itinerary (by the Leaflet routing plug-in) from the user location to the place chosen; v) the module that analyses the density of herbal plantation operates in two modes. Either as a heat map representing the density of planting is presented, or by presenting marker clustering explicitly indicating the amount of planting and coordinating with a given type of herb on a marker pinned on the map. The user may zoom in or out and also focus on different areas; vi) the data management module relies on PHP scripting and the structured query language (SQL) database system. The user can add, edit, delete and search for required data stored in the PostgreSQL database, with POSTGIS extension.

Unlike most existing applications, a general user is allowed to make changes to herbal plantations and distribution sites subject to approval from an officer or the administrative staff. Officers enjoy the same level of access as a general user but can in addition manage data fields authorized by the agency, with which they are affiliated. For examples, as stated in Table 1, a provincial agriculture officer can only manage plantation data, while an officer from the Provincial Public Health Office can access a wider degree of information. To maintain role-based functions, all system users must first login to gain their respective privileges assigned by the administrator, who can perform all functions as well as have access to the complete dataset and user management tools.

The database system was designed based on the entity relationship (ER) diagram depicted in Figure 5. The corresponding normalized schema of 10 tables (herbal; herbal product; product; herbal research; research; herbal plantation; product distribution; distribution; agriculturist; and user), their connections and subtitles are shown in the Figure. This database is used to store the data described in Table 1.

\section{System implementation}

Figure 6 shows the herbal data management screen. On this screen, a user can add an herb name, its medicinal properties, plantation and distribution sites and upload photographs. These data are, however, subject to verification by the system administrator or an authorized officer before they can be made available by the system. Once verified, uploaded photographs can be displayed as shown in Figure 7A. The frequency at which each herbal plant was accessed (a measure of the public interest in it) is shown next to an eye icon. By selecting a specific plant, the user opens a screen showing all details, such as medicinal properties, plantations, distribution sites, products and relevant research done. Lemon grass is shown as an example in Figure 7B.

Localization of plantation and distribution sites is analysed and presented on the interactive map. The data can also be augmented by descriptive and photographic information presented via the interactive map menu icon (Figure 8). In this example, data of plantations and distribution sites are represented by different colour pins. The user may call up sites by selecting herbal type or product, either one after the other or simultaneously, as well as interactively choose any pin to see the corresponding details, e.g. herb name, coordinates, address where planted and address where distributed. The user can also navigate from his or her location to the required site by means of the automatic routing function. For example, as illustrated in Figure 9, the interactive map shows the optimal route between source and destination including itinerary details (i.e. total travelling distance and time, intermediate turning points and partial distances) as suggested on the left and right panels. The distribution and density of each medicinal plantation can

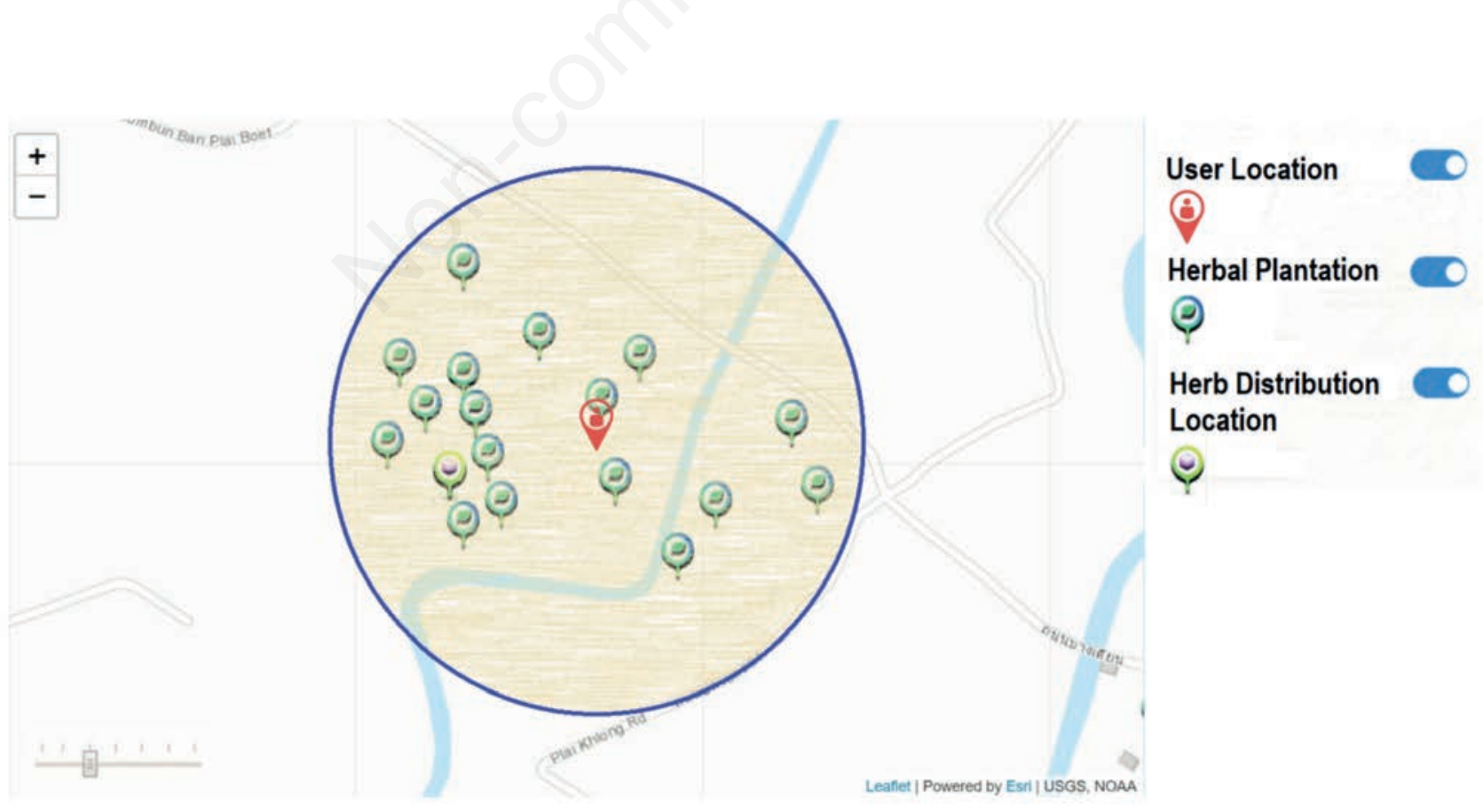

Figure 4. Interactive map showing user location, plantation and distribution site of a selected plant within a radius chosen. The user may vary the buffer radius of the circle by sliding the distance bar at the bottom left. 


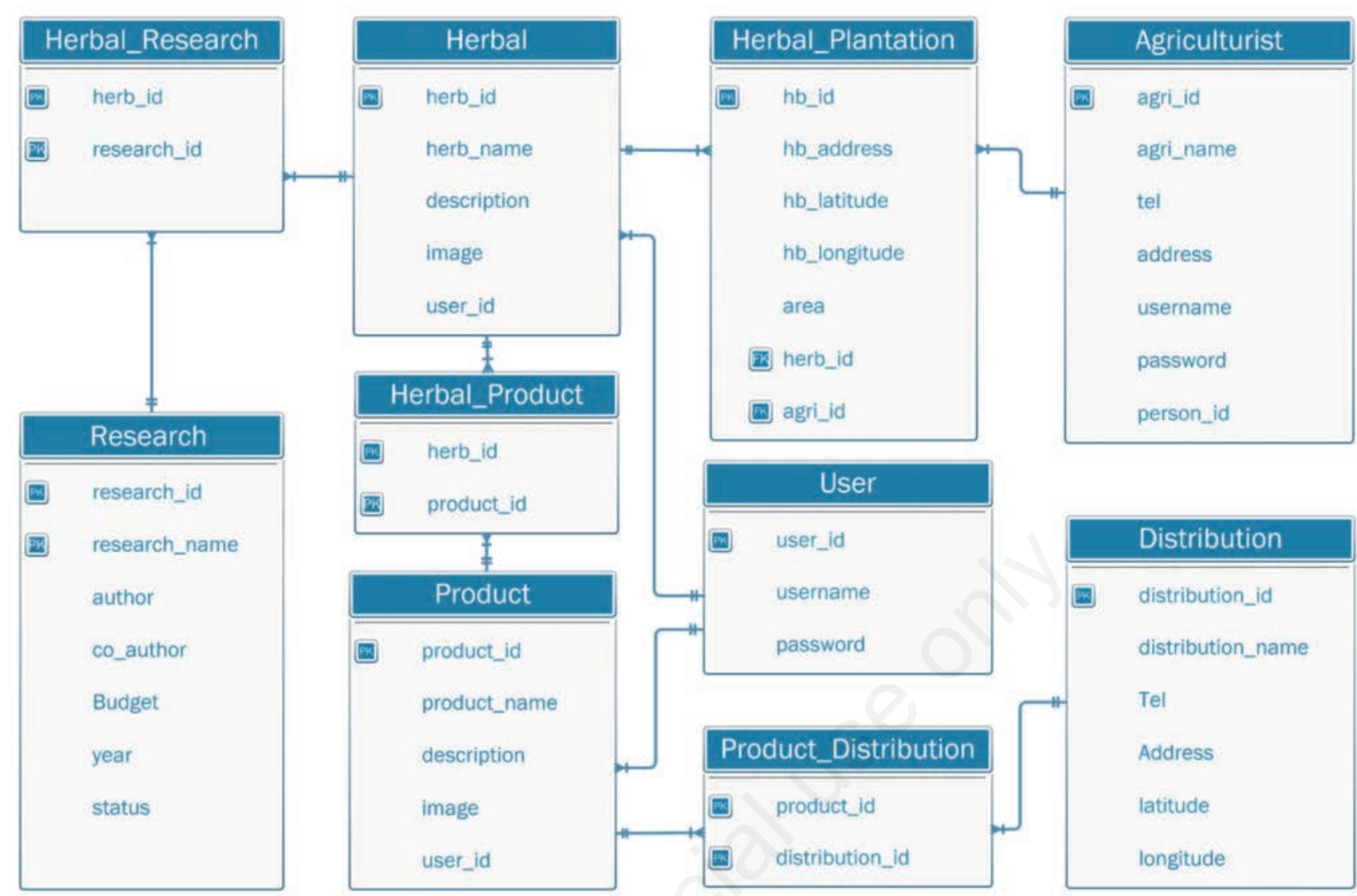

Figure 5. Diagram of the herbal plant database with related connections.

be analysed and presented as clusters or as a heat map. In the cluster mode, as shown in Figure 10A, the number of plantation plots is displayed in real time by circles of different colour indicates the size of the plantation in question. If users add or delete relevant plantation records, the actual counts will be dynamically reflected by this input. Zooming in on selected places gives further information. When choosing the heat map option for finding out about the density of herbal plantations, these are visualized by colours that indicate gradual representations of the density levels. Herbal plantations with low, medium and high densities are displayed in blue, green and red colours, respectively. Figure 11 illustrate plantation areas in Muang District and Phanom District. For example, it is evident that the area near the Ban Khao Na Nai school, exhibits densely planted herbs. Importantly, the heat map merely represents the density and does not reflect the absolute plot count. Instead, the number of plantation plots in given areas is listed in another summary report. The heat map is dynamically updated as herbal records change.

\section{Discussion}

In modern health awareness societies, medicinal plants have recently been attracting much interest from both the general public and the healthcare sectors. In response, Thai Government promotes usage of medicinal plants both in local communities and at

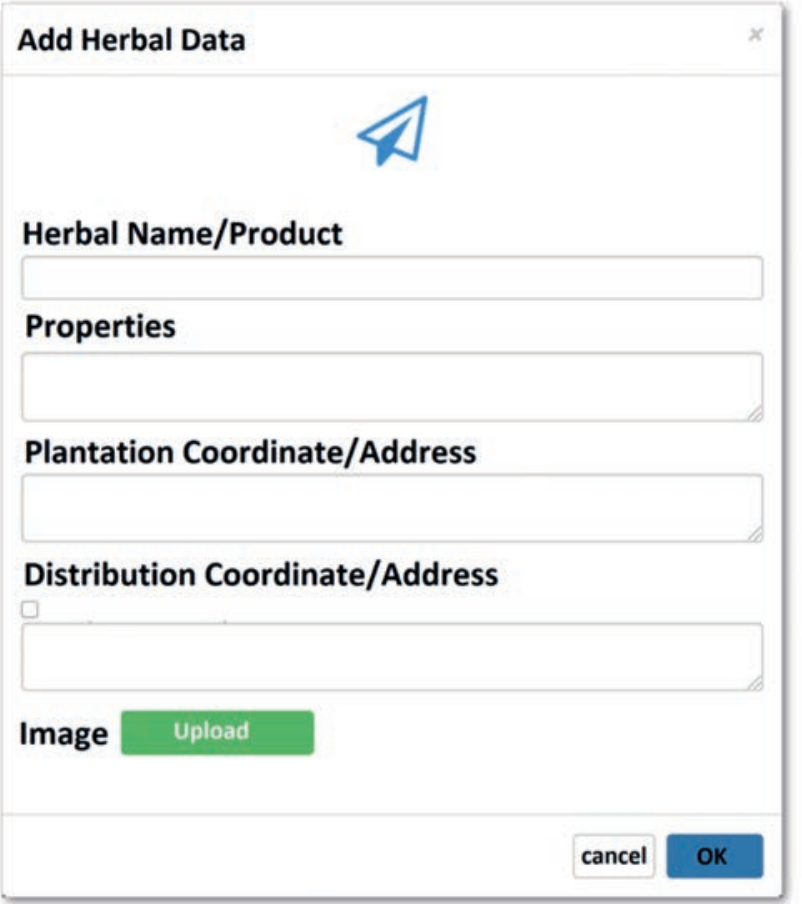

Figure 6. Input screen for herbal information. 
the industrial level. Compared to the previously developed systems for medicinal plants (Jungialern et al., 2012; Chantraket et al., 2014; Mashayekhan et al., 2016; Biswas et al., 2017), several key improvements have been made. In contrast to herbal data given verbatim, our product offers dynamic data management and inter- active real-time reports on a typical web browser. It also allows finding both attributes and spatial data, conditional retrieval and reporting. For example, herbal plantation sites and densities within a given distance to the location of a user can be displayed opening the possibility for immediate geographical access (e.g. for bulk

\section{A}

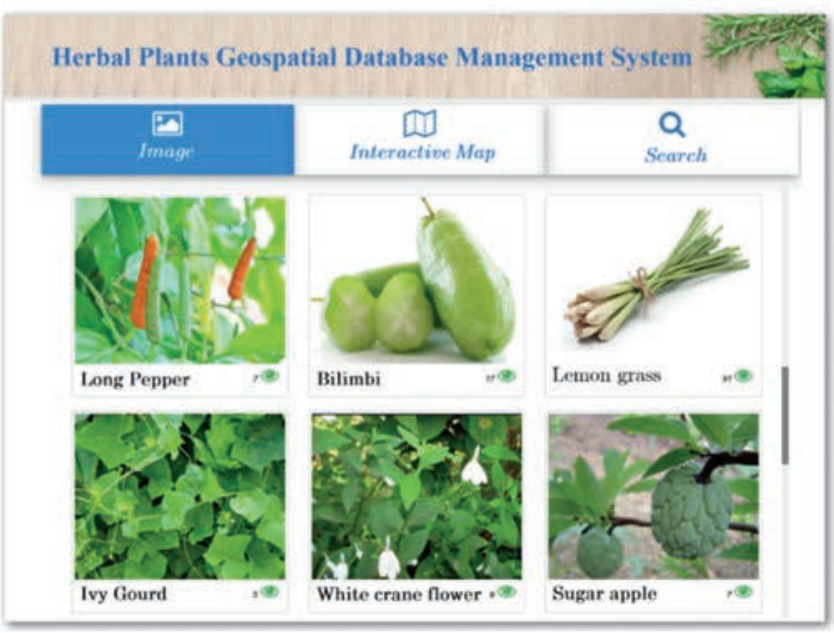

B

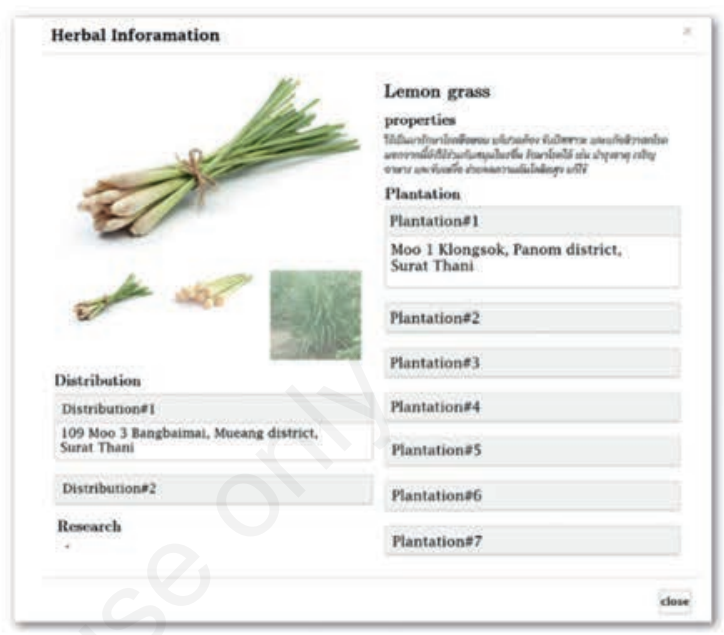

Figure 7. Page allowing selection of plants for further information: A) page with icon views of medicinal plants showing access frequencies; B) page showing example of details provided after a plant has been selected.

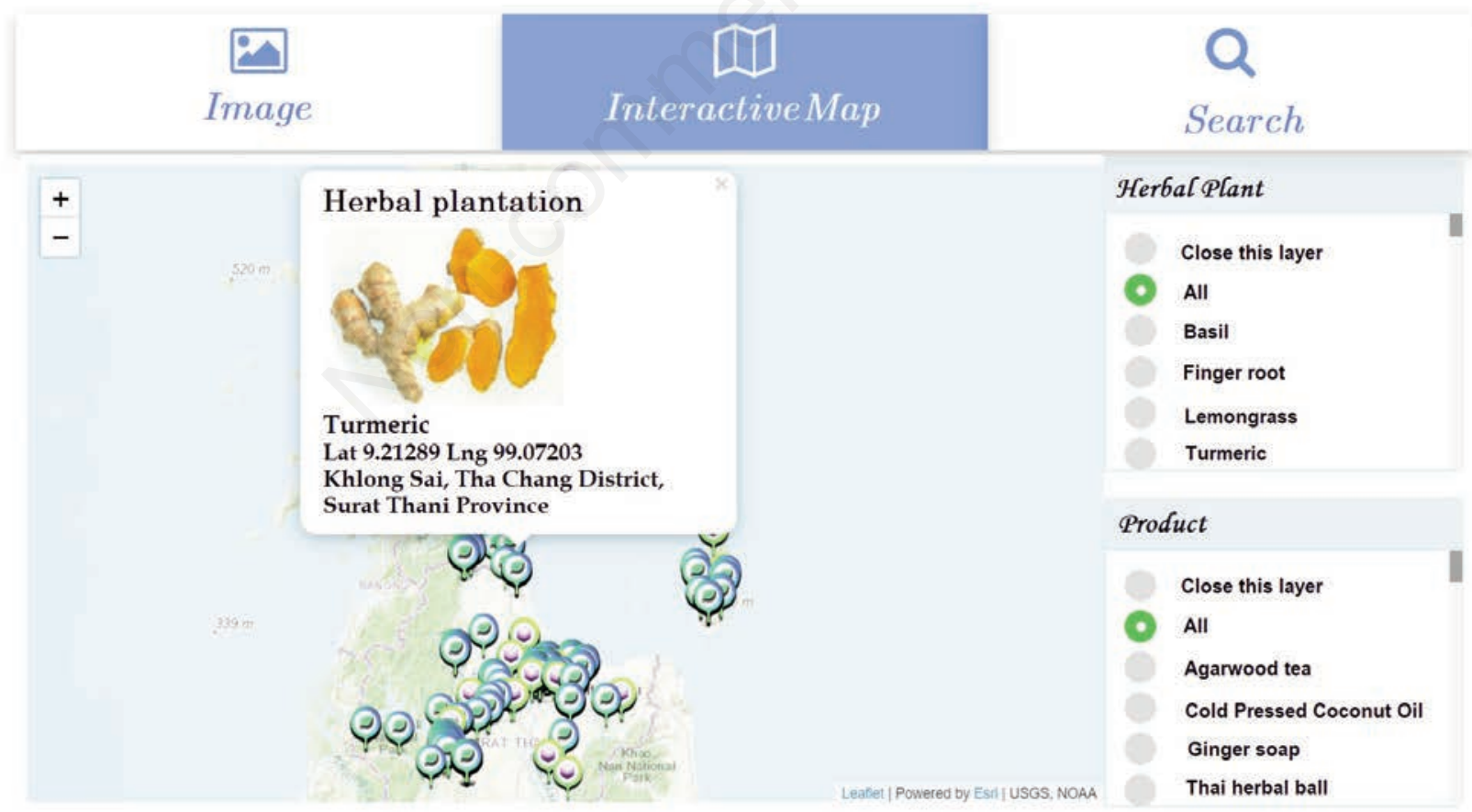

Figure 8. Interactive map giving spatial data for selected herbal plants and products. 
purchase or logistic planning) following a suggested routing. One of the major contributions of the present work is geospatial analyses and displays. Particularly, clusters and density of herbal plantations at different levels can interactively be presented without having to first import software from other packages, such as QGIS or ArcGIS. Furthermore, even if the approach suggested by AlBakri et al. (2011) early on, our graphical reports are updated in real time as soon as user input data have been approved.

As demonstrated by the results from the prototype system described, it is evident that the geospatial database and web appli-

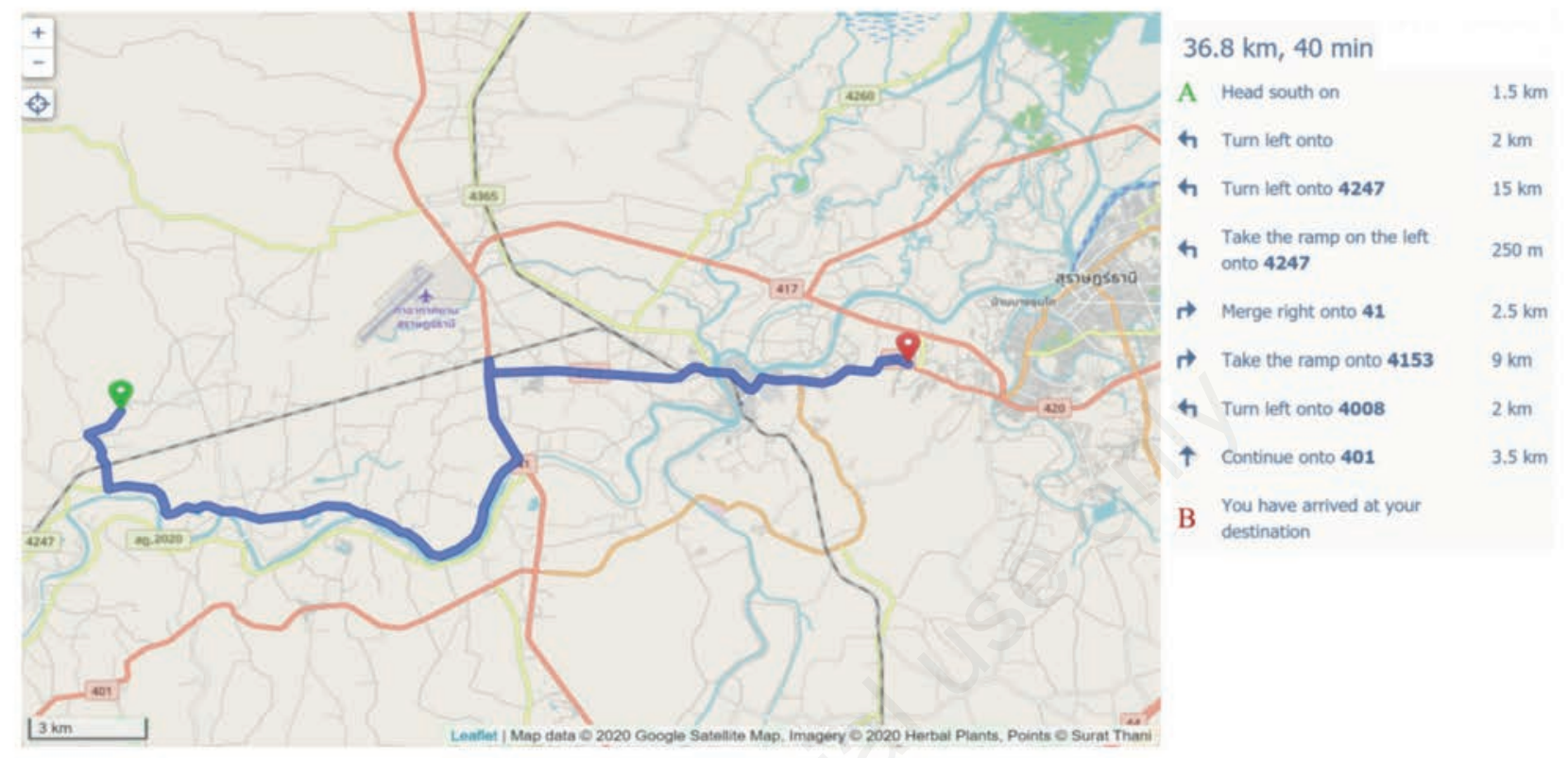

Figure 9. Automatic routing from user to a selected site.
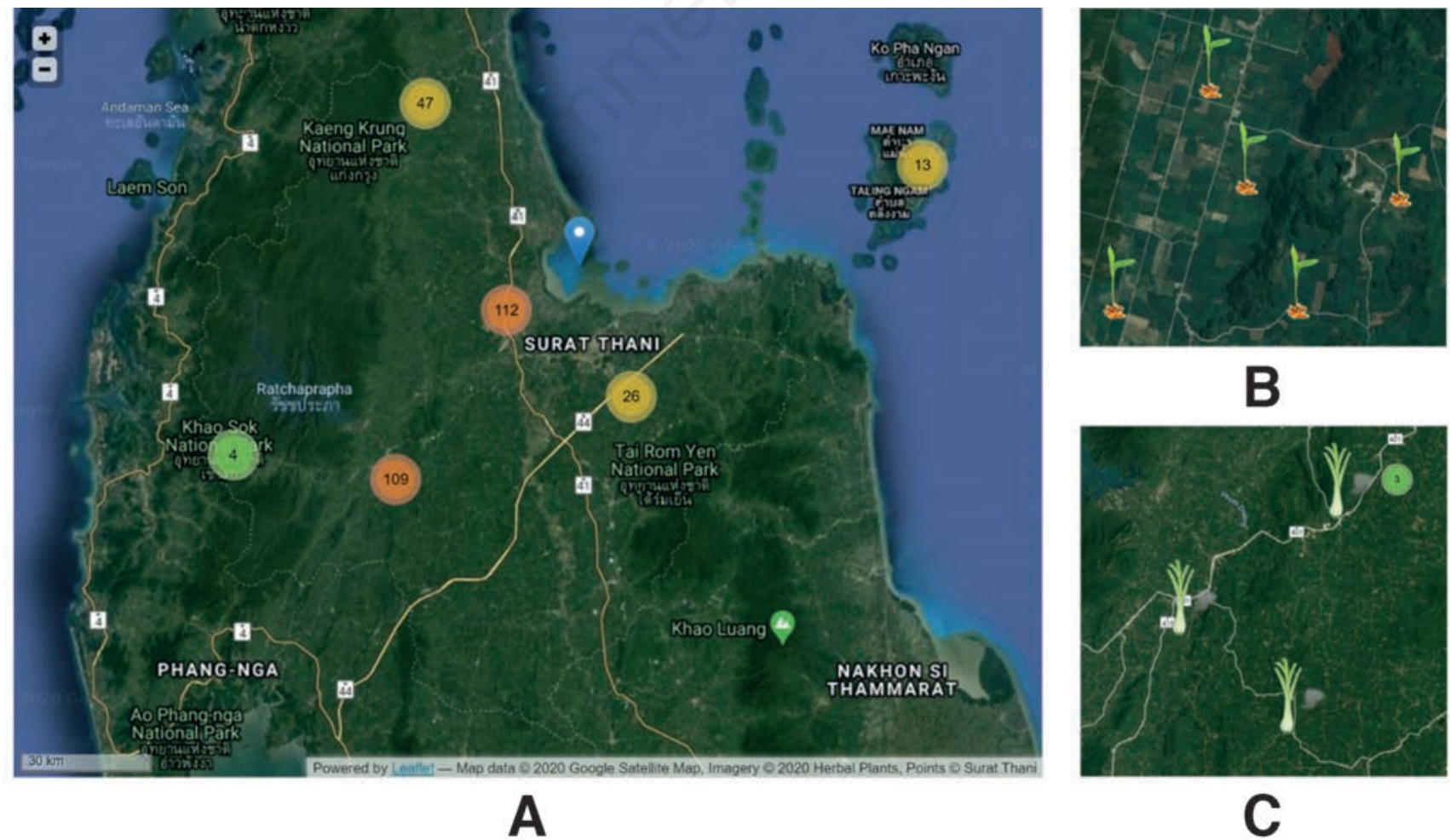

B
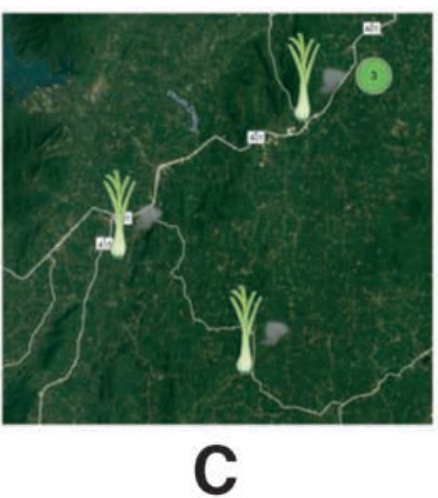

Figure 10. Distribution of a set of plantation clusters at different enlargements (A). The colour indicates the size of the plantation $<10$ plots; yellow $<100$ plots; red $\geq 100$ plots (exact number of plots given in real time in the map). Zooming in on specific locations show photographic icons of the specific herbal types planted there, e.g. as seen in (B) plots of turmeric; and in (C) plots of lemongrass. 
cation platform can find and analyse spatial data as well as present the relevant reports in real time. Thanks to cross-platform geographical JavaScript libraries and Google API, the web application offers dynamic data analyses in a similar manner as desktop software. Herbal plants data can be crowd-sourced from public participants and verified by the authorities. Subsequently, both spatial data and their attributes are published on an interactive map in real time. These functions save time and expenditure with respect to data acquisition, while Internet access makes it possible to ubiquitously present useful information, including logistics to nearby plantations and distribution sites. Naturally, the presentations described can be generalized, i.e. data management, analyses and interactive reports can as well be applied to other regions (domestic and international), as they are by no means limited by the GIS functions. However, further research directions will be needed to also consider climate variables and environmental factors as well as logistics and potential customers in determining the most suitable areas for planting given herbs.
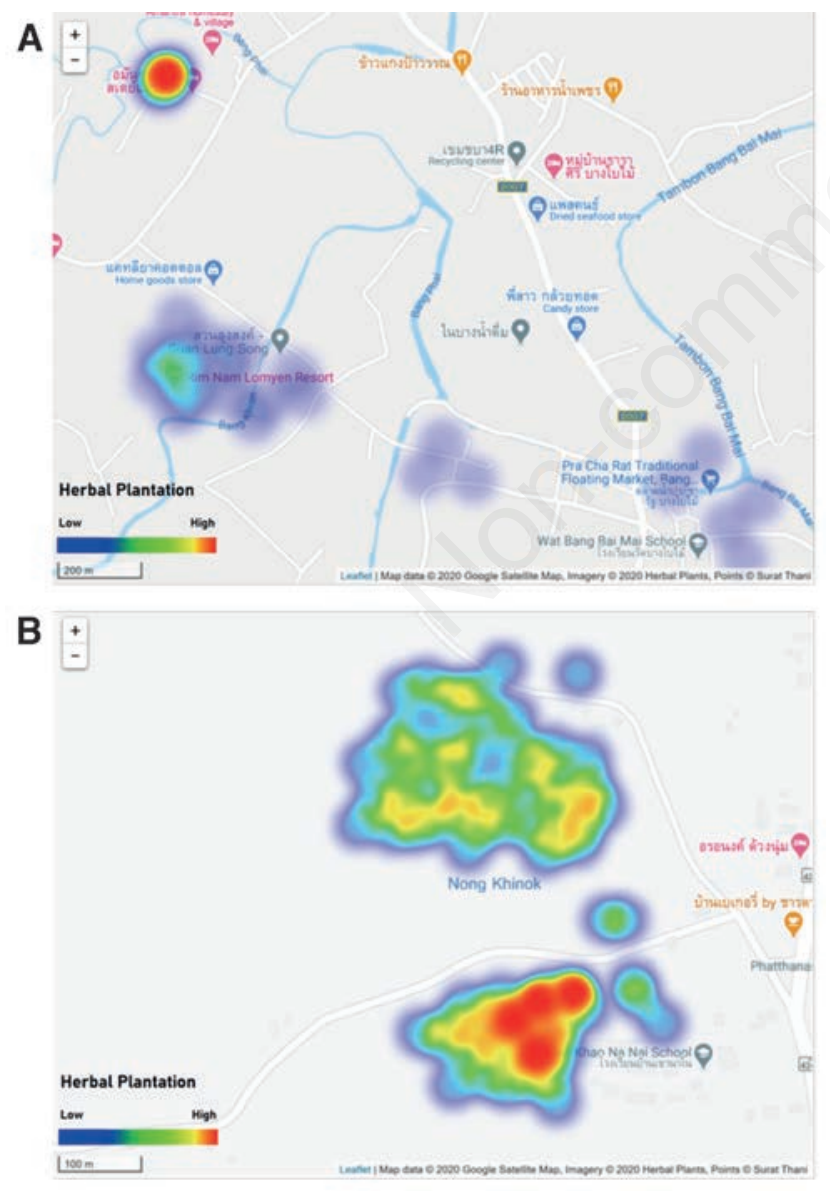

Figure 11. Heat map representation of the plant density in two areas with herbal plantations. A) Muang District; B) Phanom District.

\section{Conclusions}

A new, computer-based system has been developed offering an effective means of collaboration, among herb farmers, government agencies, private investors, healthcare professionals and general customers on various aspects of medicinal plants and their applications. The resultant reports are useful for supporting decision-making, agro-economic and agro-ecological planning and devising public policies on these plants. Based on public GIS data repositories via Google API and cross-platform JavaScript libraries, the developed system could be extended to other regions and include a wider range of herbal types.

\section{References}

Al-Bakri JT, Al-Eisawi D, Damhoureyeh S, Oran S, 2011. GISbased analysis of spatial distribution of medicinal and herbal plants in arid and semi-arid zones in the Northwest of Jordan. Ann Arid Zone 50:99-115.

Biswas B, Walker S, Varun M, 2017. Web GIS based identification and mapping of medicinal plants: a case study of Agra (up), India. Plant Archives 17:8-20.

Bultum LE, Woyessa AM, Lee D, 2019. ETM-DB: integrated Ethiopian traditional herbal medicine and phytochemicals database. BMC Complem Altern Med 19:212.

Chantraket R, Uttaruk P, Skole DL, Samek JH, Castaneda O, 2014. Internet-based development of medicinal plants geospatial system in Phu Phan forest complex: northeastern Thailand. Agric Nat Resour 48:227-40.

Chotchoungchatchai S, Saralamp P, Jenjittikul T, Pornsiripongse S, Prathanturarug S, 2012. Medicinal plants used with Thai Traditional Medicine in modern healthcare services: A case study in Kabchoeng Hospital, Surin Province, Thailand. J Ethnopharmacol 141:193-205.

Fausat OB, Olayinka TA, 2019. Design and implementation of herbal therapy knowledge management system (HTKMS). Science 7:44-52.

Jokar NK, Noorhosseini SA, Allahyari MS, Damalas CA, 2017. Consumers' acceptance of medicinal herbs: an application of the technology acceptance model (TAM). J Ethnopharmacol 207:203-10.

Khaerunnisa S, Kurniawan H, Awaluddin R, Suhartati S, Soetjipto S, 2020. Potential inhibitor of COVID-19 main protease (Mpro) from several medicinal plant compounds by molecular docking study. Preprints 2020:2020030226. doi: 10.20944/preprints202003.0226.v1.

Kolasinski SL, 2014. Food, drink, and herbs: alternative therapies and gout. Curr Rheumatol Rep 16:409.

Mary JA, Priyadharshini KC, Amal GPR, Ramya G, Nithya R, Ambika MB, Shenbagarathai R, 2012. MEDDB: A medicinal plant database developed with the information gathered from tribal people in and around Madurai, Tamil Nadu. Bioinformation 8:391.

Mashayekhan A, Pourmajidian MR, Jalilvand H, Gholami MR, Teimouri MS, 2016. Economic importance and GIS mapping of medicinal plants in Iran: a case study of Darkesh. J Appl Sci Environ Manage 20:646-50.

Mason R, 2002. Herbal medicine/phytotherapy databases. Altern Complement Ther 8:127-8.

Ochwang'i DO, Oduma JA, 2017. Overview of governmental sup- 
port across Africa toward the development and growth of herbal medicine. In: Kuete V (Ed.), Medicinal spices and vegetables from Africa. Academic Press, New York, NY, USA, pp 153-169.

Ogirima SA, 2015. Web-based decision support system for prescription in herbal medicine. J Emerg Trends Engine Applied Sci 6:245-54.

Saxton JD, Owen DJ, 2005. Developing optimal search strategies for finding information on herbs and other medicinal plants in MEDLINE®. J Alternat Complement Med 11:725-31.

Serafini M, Peluso I, 2016. Functional foods for health: the interrelated antioxidant and anti-inflammatory role of fruits, vegetables, herbs, spices and cocoa in humans. Curr Pharma Design 22:6701-15.
Tungkwampian W, Theerarungchaisri A, Buranarach M, 2015. Development Thai herbal medicine knowledge base using ontology technique. Thai J Pharma Sci (TJPS) 39:3.

Vetrivel U, Subramanian N, Pilla K, 2009. InPACdb Indian plant anticancer compounds database. Bioinformation 4:71.

Welz AN, Emberger-Klein A, Menrad K, 2018. Why people use herbal medicine: insights from a focus-group study in Germany. BMC Complement Alternat Med 18:92.

Wootton JC, 2002. Development of HerbMed®: An interactive, evidence-based herbal database. Adv Phytomed 1:55-60.

Wu J, Li X, Huang L, Meng X, Hu H, Luo L, Chen S, 2019. A new GIS model for ecologically suitable distributions of medicinal plants. Chinese Med 14:4. 